\title{
The novel sodium channel modulator GS-458967 (GS967) is an effective treatment in a mouse model of $S C N 8 A$ encephalopathy
}

\author{
Erin M. Baker ${ }^{1}$ | Christopher H. Thompson ${ }^{1}$ | Nicole A. Hawkins ${ }^{1}$ | Jacy L. Wagnon ${ }^{2}$ | \\ Eric R. Wengert ${ }^{3}$ | Manoj K. Patel ${ }^{3}$ | Alfred L. George Jr. ${ }^{1}$ | Miriam H. Meisler ${ }^{2}$ | \\ Jennifer A. Kearney ${ }^{1}$
}

${ }^{1}$ Department of Pharmacology, Northwestern University Feinberg School of Medicine, Chicago, IL, USA

${ }^{2}$ Department of Human Genetics, University of Michigan, Ann Arbor, MI, USA

${ }^{3}$ Department of Anesthesiology, University of Virginia Health System, Charlottesville, VA, USA

\section{Correspondence}

Jennifer A. Kearney, Department of Pharmacology, Northwestern University Feinberg School of Medicine, Chicago, IL, USA.

Email: jennifer.kearney@northwestern.edu

\section{Funding information}

Feinberg School of Medicine; National Institute of Neurological Disorders and Stroke, Grant/Award Number: R01 NS103090; PhRMA Foundation

\begin{abstract}
Summary
Objective: De novo mutations of $S C N 8 A$, encoding the voltage-gated sodium channel $\mathrm{Na}_{\mathrm{V}} 1.6$, have been associated with a severe infant onset epileptic encephalopathy. Individuals with $S C N 8 A$ encephalopathy have a mean age of seizure onset of 4-5 months, with multiple seizure types that are often refractory to treatment with available drugs. Anecdotal reports suggest that high-dose phenytoin is effective for some patients, but there are associated adverse effects and potential for toxicity. Functional characterization of several SCN8A encephalopathy variants has shown that elevated persistent sodium current is one of several common biophysical defects. Therefore, specifically targeting elevated persistent current may be a useful therapeutic strategy in some cases.
\end{abstract}

Methods: The novel sodium channel modulator GS967 has greater preference for persistent as opposed to peak current and nearly 10-fold greater potency than phenytoin. We evaluated the therapeutic effect of GS967 in the Scn $8 a^{N 1768 D /+}$ mouse model carrying an SCN8A patient mutation that results in elevated persistent sodium current. We also performed patch clamp recordings to assess the effect of GS967 on peak and persistent sodium current and excitability in hippocampal neurons from $S c n 8 a^{N 1768 D /+}$ mice.

Results: GS967 potently blocked persistent sodium current without affecting peak current, normalized action potential morphology, and attenuated excitability in neurons from heterozygous Scn $8 a^{N 1768 D^{/+}}$mice. Acute treatment with GS967 provided dose-dependent protection against maximal electroshock-induced seizures in Scn $8 a^{N 1768 D /+}$ and wild-type mice. Chronic treatment of Scn8 $8 a^{N 1768 D /+}$ mice with GS967 resulted in lower seizure burden and complete protection from seizure-associated lethality observed in untreated $S c n 8 a^{N 1768 D /+}$ mice. Protection was achieved at a chronic dose that did not cause overt behavioral toxicity or sedation.

Significance: Persistent sodium current modulators like GS967 may be an effective precision targeting strategy for SCN8A encephalopathy and other functionally similar channelopathies when elevated persistent sodium current is the primary dysfunction.

\section{K E Y W O R D S}

epilepsy, epileptic encephalopathy, mouse model, pharmacology, voltage-gated sodium channel 


\section{INTRODUCTION}

Epilepsy is a common neurological disorder, with a lifetime incidence of 1 in $26 .{ }^{1}$ Mutations in genes encoding neuronal voltage-gated sodium channels have been implicated in several types of human epilepsy, including epileptic encephalopathies. ${ }^{2}$ These are severe epilepsies with cooccurring cognitive, behavioral, and neurological deficits due to the combined effect of seizure activity and the underlying mutation. ${ }^{3}$ In recent years, $>150$ cases of epileptic encephalopathy have been associated with de novo mutations in $S C N 8 A$, encoding the $\mathrm{Na}_{\mathrm{V}} 1.6$ voltage-gated sodium channel pore-forming (alpha) subunit. The clinical phenotype begins in the first year of life with pleiomorphic seizures that are difficult to control, delayed cognitive development, and motor impairment. ${ }^{4}$ SCN8A encephalopathy is also associated with an elevated risk for sudden unexpected death in epilepsy (SUDEP), making seizure control a high priority. ${ }^{5,6}$

The first report of SCN8A encephalopathy described a female proband with multiple seizure types, intellectual disability, developmental delay, and motor impairment. ${ }^{2}$ She succumbed to SUDEP at 15 years of age. Whole genome sequencing of the family quartet identified the heterozygous, de novo mutation SCN8A p.Asn1768Asp (N1768D). ${ }^{7}$ Electrophysiologic recording of sodium currents in ND7/23 cells expressing the mutant channel revealed a large persistent sodium current as the primary biophysical defect, as well as incomplete channel inactivation and a shift in the voltage dependence of steady-state fast inactivation. ${ }^{7}$ Introduction of the N1768D mutation into the murine Scn8a gene resulted in a mouse model that recapitulates many features of the human syndrome. $^{8,9}$ Specifically, heterozygous Scn $8 a^{N 1768 D /+}$ $\left(S c n 8 a^{D /+}\right)$ mice exhibit spontaneous seizures, ataxia, cardiac arrhythmia, and premature death, although the age of seizure onset at 2-4 months is late relative to the $S C N 8 A^{N 1768 D}$ proband. Homozygous Scn8a $a^{N 1768 D / N 1768 D}\left(\operatorname{Scn} 8 a^{D / D}\right)$ mice exhibit these features with earlier onset and accelerated progression. Similar to recordings in heterologous expression systems, neurons from $\operatorname{Scn} 8 a^{D /+}$ mice have elevated persistent current and higher spontaneous firing rate compared to wild-type (WT) neurons. ${ }^{7,10}$ The mouse model enables preclinical evaluation of potential anticonvulsant treatments for SCN8A encephalopathy.

Several types of biophysical defects have been documented for variants associated with SCN8A encephalopathy. Targeting persistent sodium current, one of the common primary biophysical defects in this disorder, may provide an effective precision therapeutic approach. Reports have described patients achieving seizure control with carbamazepine, oxcarbazepine, valproic acid, and most notably, high-dose phenytoin. ${ }^{11-16}$ Phenytoin is a state-

\section{Key Points}

- GS967 potently suppressed persistent sodium current in acutely dissociated neurons and hyperexcitability in slices from heterozygous $\operatorname{Scn} 8 a^{N 1768 D /+}$ mice

- Acute treatment with GS967 dose-dependently protected both $S c n 8 a^{N 1768 D /+}$ and wild-type mice against MES-induced seizures

- Chronic GS967 treatment was associated with significantly lower spontaneous seizure burden and completely protected $\operatorname{Scn} 8 a^{N 1768 D /+}$ mice from seizure-associated lethality

- Chronic GS967 did not cause overt behavioral toxicity or sedation in wild-type mice at the effective anticonvulsant dose

dependent sodium channel blocker with a small preference $(\sim 10 \%)$ for persistent current over peak current. ${ }^{17}$ However, high-dose phenytoin has significant safety concerns due to saturable metabolism and risk for phenytoin poisoning. ${ }^{18-21}$ GS-458967 (GS967) is a novel sodium channel modulator specifically developed to inhibit persistent sodium current, with a 42-fold preference for persistent as opposed to peak current inhibition. ${ }^{22}$ Our previous studies demonstrated that acute application of GS967 to hippocampal neurons did not affect the current-voltage relationship or voltage dependence of activation, but induced a hyperpolarized shift of steady-state channel inactivation and slowed both recovery from fast inactivation and onset of slow inactivation. ${ }^{23}$ GS967 has also been reported to have strong use-dependent block. $^{24}$

GS967 was previously tested in the Scn2a 254 mouse model with epilepsy caused by elevated persistent sodium current in hippocampal neurons. ${ }^{25}$ Acute application of GS967 to neurons isolated from untreated Scn2a 254 mice reduced persistent sodium current and spontaneous firing, and GS967 treatment of Scn2a $a^{Q 54}$ mice reduced seizure burden and improved survival. Considering the etiological similarities between $S c n 2 a^{Q 54}$ and $S c n 8 a^{D /+}$ mice, we hypothesized that treatment with GS967 may have a therapeutic benefit in $S c n 8 a^{D /+}$ and $S c n 8 a^{D / D}$ mice.

In the current study, we tested the effect of GS967 on persistent sodium current and action potential firing in neurons, as well as seizure susceptibility, spontaneous seizures, and survival of $S c n 8 a^{D /+}$ mice. We report that GS967 suppresses persistent sodium current and excitability in neurons ex vivo, and protects against seizures and premature lethality in vivo, supporting targeting of persistent sodium current as a potential therapeutic strategy for this disorder. 


\section{2 | MATERIALS AND METHODS}

\section{1 | Mice}

Scn $8 a^{e m 1 M m}$ mice were generated using TALEN technology and previously characterized. ${ }^{8,9}$ The line was made congenic by continuous backcrossing to $\mathrm{C} 3 \mathrm{HeB} / \mathrm{FeJ}$ WT mice (stock \# 000658; Jackson Laboratory, Bar Harbor, ME, USA) for $>10$ generations. Mice were genotyped by polymerase chain reaction of tail biopsy DNA as previously described. ${ }^{8}$ Heterozygous experimental mice were obtained by breeding $S c n 8 a^{D /+}$ males with $\mathrm{C} 3 \mathrm{HeB} / \mathrm{FeJ}$ females. Homozygous experimental mice were generated by breeding $S c n 8 a^{D /+}$ males with $S c n 8 a^{D /+}$ females. Mice were housed in a specific pathogen-free barrier facility with a 14/10-hour light/dark cycle and access to food and water ad libitum. Animal studies were approved by the Northwestern University and University of Virginia animal care and use committees in accordance with the National Institutes of Health Guide for the Care and Use of Animals. Principles outlined in the ARRIVE (Animal Research: Reporting of In Vivo Experiments) and Basel declarations (including the 3R concept) were considered in experimental design. Both male and female mice were used in all experiments.

\section{2 | Drugs}

GS967 was provided by Gilead Sciences (Foster City, CA, USA) or purchased from Cayman Chemical (Ann Arbor, MI, USA). Phenytoin sodium solution was manufactured by WestWard Pharmaceuticals (Eatontown, NJ, USA) and obtained from Patterson Veterinary Supply (Greeley, CO, USA). Phenytoin for electrophysiologic experiments was obtained from Sigma-Aldrich (St. Louis, MO, USA). Drug solutions for electrophysiologic experiments were prepared in dimethylsulfoxide. For acute administration to mice in maximal electroshock (MES) experiments, GS967 was solubilized in vegetable oil, and phenytoin sodium solution was diluted with $0.5 \%$ methyl cellulose in water. Vehicle control mice were administered either vegetable oil or $0.5 \%$ methyl cellulose in water. For chronic administration studies, mice were fed Purina 5001 rodent chow compounded with GS967 (8 mg/kg of chow; Research Diets, New Brunswick, NJ, USA). ${ }^{23,25}$ The estimated dose was $1.5 \mathrm{mg} / \mathrm{kg} / \mathrm{d}$ based on average daily consumption of $190 \mathrm{~g}$ of chow/kg of body weight (http://www. researchdiets.com/resource-center-page/typical-food-intake). Previous studies showed that chronic treatment with this dose resulted in GS967 plasma and brain concentrations of $1.0 \pm 0.08$ and $1.7 \pm 0.1 \mu \mathrm{mol} \cdot \mathrm{L}^{-1}$, respectively. ${ }^{23}$ Chronic treatment with GS967 at $1.5 \mathrm{mg} / \mathrm{kg} / \mathrm{d}$ did not cause overt adverse neurobehavioral or sedating effects in WT $\mathrm{C} 3 \mathrm{HeB} / \mathrm{FeJ}$ at 5-7 weeks of treatment (Figure S1).

\section{3 | Isolated neuron preparation and electrophysiologic recording}

Neurons were acutely isolated from untreated $\operatorname{Scn} 8 a^{D /+}$ mice and WT littermates between 30 and 35 days of age. Hippocampal neurons were isolated as previously described. $^{25}$ Whole-cell voltage clamp experiments of acutely isolated pyramidal neurons were performed at room temperature using previously described methods. ${ }^{26,27}$ External recording solution contained (in $\mathrm{mmol} \cdot \mathrm{L}^{-1}$ ): $20 \mathrm{NaCl}$, $100 \mathrm{~N}$-methyl-D-glucamine, 10 hydroxyethylpiperazine ethane sulfonic acid (HEPES), $1.8 \mathrm{CaCl}_{2} \cdot 2 \mathrm{H}_{2} \mathrm{O}, \quad 2$ $\mathrm{MgCl}_{2} \cdot 6 \mathrm{H}_{2} \mathrm{O}$, and 20 tetraethylammonium chloride, with $\mathrm{pH}$ adjusted to 7.35 with $\mathrm{HCl}$ and osmolarity adjusted to $310 \mathrm{mOsm} / \mathrm{L}$ with sucrose. Internal solution consisted of (in $\mathrm{mmol} \cdot \mathrm{L}^{-1}$ ): $5 \mathrm{NaF}, 105 \mathrm{CsF}, 20 \mathrm{CsCl}, 2$ ethyleneglycoltetraacetic acid (EGTA), and 10 HEPES, with $\mathrm{pH}$ adjusted to 7.35 with $\mathrm{CsOH}$ and osmolarity adjusted to $280 \mathrm{mOsmol} / \mathrm{kg}$ with sucrose. All whole-cell voltage clamp recordings utilized a holding potential of $-120 \mathrm{mV}$. Voltage-gated sodium currents were recorded in the absence and presence of $500 \mathrm{nmol} \cdot \mathrm{L}^{-1}$ tetrodotoxin, and analyzed following offline digital subtraction, as previously described. $^{26,27}$ Persistent current was measured utilizing a 200-millisecond depolarizing step. Mean current in the final 10 milliseconds was normalized to peak transient current. Statistical comparisons were made using Student's $t$ test or 1-way analysis of variance (ANOVA) followed by Tukey post hoc test, and $P<.05$ was considered statistically significant.

\section{4 | Brain slice preparation and electrophysiologic recording}

Brain slices were prepared from WT and $S c n 8 a^{D /+}$ mice (>8 weeks of age) using a Leica Microsystems (Wetzlar, Germany) VT1200 vibratome as previously described. ${ }^{10}$ CA1 pyramidal neurons were visually identified using infrared video microscopy (Hamamatsu Photonics, Hamamatsu, Japan) with a Zeiss (Oberkochen, Germany) Axioscope microscope. Whole-cell current clamp recordings were performed as previously described. ${ }^{10}$ During recordings, slices were held in a small chamber and superfused with oxygenated artificial cerebrospinal fluid (in $\mathrm{mmol} \cdot \mathrm{L}^{-1}$ : $125 \mathrm{NaCl}, 2.5 \mathrm{KCl}, 1.25 \mathrm{NaH}_{2} \mathrm{PO}_{4}, 2 \mathrm{CaCl}_{2}, 1 \mathrm{MgCl}_{2}$, 0.5 L-ascorbic acid, 10 glucose, $25 \mathrm{NaHCO}_{3}$, and 2 sodium pyruvate [osmolarity $=300-312 \mathrm{mOsm} / \mathrm{L}$ ]) at $\sim 28^{\circ} \mathrm{C}$ at a rate of $1-2 \mathrm{~mL} / \mathrm{min}$. Intracellular recording solution contained (in mmol. $\mathrm{L}^{-1}$ ): $120 \mathrm{~K}$-gluconate, $10 \mathrm{NaCl}, 2 \mathrm{MgCl}_{2}$, $0.5 \mathrm{~K}_{2}$ EGTA, 10 HEPES, $4 \mathrm{Na}_{2} \mathrm{ATP}$, and $0.3 \mathrm{NaGTP}$, and $\mathrm{pH}$ was adjusted to 7.2 with $\mathrm{KOH}$ (osmolarity $=270$ $290 \mathrm{mOsm} / \mathrm{L}$ ). 
Action potentials (APs) were evoked using a depolarizing current injection step of $400 \mathrm{pA}$. To standardize across neurons, resting membrane potentials were maintained at $-70 \mathrm{mV}$ by injection of direct current. Only depolarizing events that consisted of a single observable peak initiating from threshold and had an upstroke velocity $>150 \mathrm{mV} / \mathrm{ms}$ were considered as APs. Early afterdepolarizations (EADs) were identified as spikelets that occurred during the repolarizing phases of the initial AP and had slower upstroke velocities $(>15 \mathrm{mV} / \mathrm{ms})$, longer widths, and depolarized thresholds. Input resistance was calculated using a $-20-\mathrm{pA}$ hyperpolarizing current injection step. The approximate rheobase for each cell was defined as the highest current injection step that did not evoke any APs. AP duration (APD) was defined as the width of the AP at the halfway voltage between threshold and AP peak and was measured for the first AP elicited during a 400-pA current injection. To measure intrinsic membrane and AP properties, a ramp of depolarizing current (0-400 pA) was injected into each neuron over a 4-second time period. Threshold was identified as the membrane potential at which the upstroke velocity was $5 \%$ of its maximum. ${ }^{28} \mathrm{AP}$ amplitude, upstroke velocity, and downstroke velocity were calculated using the first evoked AP during the current ramp. Amplitude was defined as the difference between threshold and AP peak, whereas upstroke velocity and downstroke velocity were defined as the maximum and minimum slope of the evoked AP, respectively.

\section{5 | MES}

At 4 weeks of age, mice were assigned to treatment groups by block randomization and received an intraperitoneal injection of test compound (phenytoin or GS967) or its respective vehicle in a volume of $10 \mathrm{~mL} / \mathrm{kg}$ body weight 2 hours prior to testing. MES tests were conducted at the previously determined time to peak effect for GS967 and phenytoin. ${ }^{25}$ Corneal electroshock was delivered using a pulse generator set at a frequency of $60 \mathrm{~Hz}$ and 0.5 -millisecond pulse width (Ugo Basile, Varese, Italy) with shock duration $=0.2$ second and current $=20 \mathrm{~mA}(120 \mathrm{mC})$ for $S c n 8 a^{D /+}$ and $0.4 \mathrm{sec}-$ ond, $60 \mathrm{~mA}(720 \mathrm{mC})$ for WT mice. Mice were scored for the presence or absence of full tonic hindlimb extension (hindlimbs at a $180^{\circ}$ angle to torso) occurring within 5 seconds of the shock. $\mathrm{ED}_{50}$ values were determined from doseresponse curves generated and compared with Prism software (GraphPad Software, La Jolla, CA, USA).

\section{6 | Survival}

At 6 weeks of age, heterozygous $S c n 8 a^{D /+}$ mice were assigned to either GS967 or control treatment groups by block randomization. Mice in the GS967 treatment group were provided chow containing GS967 ( $8 \mathrm{mg} / \mathrm{kg}$ of chow; Research Diets). The estimated dose was $1.5 \mathrm{mg} / \mathrm{kg} / \mathrm{d}$ based on average consumption of $190 \mathrm{~g}$ of chow per $\mathrm{kg}$ of body weight. Previous studies established that this dose results in plasma levels of $\sim 1 \mu \mathrm{mol} \cdot \mathrm{L}^{-1} \cdot{ }^{23,25}$ Survival was monitored up to 9 months of age by performing census checks 3-4 days per week.

Homozygous $\operatorname{Scn} 8 a^{D / D}$ mice were generated by crossing $S c n 8 a^{D /+}$ males and $S c n 8 a^{D /+}$ females. When the resulting offspring were 5 days of age, the lactating dam was either maintained on control chow or switched to GS967 chow. Previous studies showed that GS967 passes freely to nursing pups, resulting in a plasma concentration of $\sim 1 \mu \mathrm{mol} \cdot \mathrm{L}^{-1}{ }^{23}$ Survival of the $\operatorname{Scn} 8 a^{D / D}$ pups was monitored by performing census checks 6-7 days per week.

Survival was assessed with Kaplan-Meier analysis and compared between groups using the Mantel-Cox log-rank test with $P<.05$ considered statistically significant.

\section{7 | Video-electroencephalography}

At 6 weeks of age, $S c n 8 a^{D /+}$ mice and WT littermates were assigned to either GS967 or control treatment groups by block randomization. At age 70-80 days, mice were anesthetized with isoflurane and fitted with a prefabricated head mount (Pinnacle Technology, Lawrence, KS, USA). Following at least 48 hours of recovery, mice underwent continuous video-electroencephalographic (EEG) recording for 5-13 days. Additional days of continuous video-only recording were obtained (range $=3-26$ days). Digitized EEG data were acquired and analyzed with Sirenia software (Pinnacle Technology). Epileptiform activity was scored offline by a blinded observer to identify electrographic seizures and behavioral correlates. The behavioral component of seizures scored by video-only were indistinguishable from those scored by epileptiform activity. Seizure frequencies were calculated for each individual animal and compared between treated and untreated $S c n 8 a^{D /+}$ mice using a Mann-Whitney test with $P<.05$ considered statistically significant.

\section{8 $\mid$ Central nervous system safety screen}

At 6 weeks of age, WT $\mathrm{C} 3 \mathrm{HeB} / \mathrm{FeJ}$ mice were assigned to either GS967 or control treatment groups by block randomization and commenced treatment. At 11-13 weeks of age, mice were tested on 3 assays on consecutive days using a modified Irwin screen (day 1), ${ }^{29}$ open field test (day 2), and rotarod test (day 3). All testing was conducted between 9:00 AM and 1:00 PM. For the open field assay, mice were placed in a new environment and tracked for 10 minutes (LimeLight software; Actimetrics, Wilmette, IL, USA). Mice were scored on total distance traveled. For the rotarod 
assay, mice underwent a training trial $(4 \mathrm{rpm})$ and then 3 test trials with acceleration from 4 to $40 \mathrm{rpm}$ over $5 \mathrm{~min}$ (Panlab/Harvard Apparatus, Holliston, MA, USA). Trials were separated by 15 minutes of rest. Latency to fall was recorded for the 3 trials and averaged. Parametric data were compared using Student's $t$ tests, and nonparametric data were compared using Mann-Whitney $U$ test, with $P<.05$ considered statistically significant. No significant sex differences were observed, so groups were collapsed across sex.

\section{3 | RESULTS}

\subsection{GS967 suppresses persistent current in hippocampal neurons from $S c n 8 a^{D /+}$ mice}

Using whole-cell voltage clamp recording, we measured persistent sodium current in acutely isolated hippocampal pyramidal neurons from postnatal day 30-35 (P30-P35) $\operatorname{Scn} 8 a^{D /+}$ mice. Persistent current was assessed as tetrodotoxin-sensitive current following a 200-millisecond depolarization. In untreated neurons from $S c n 8 a^{D /+}$ mice, persistent current was equal to $1.7 \pm 0.3 \%$ of peak current ( $\mathrm{n}=8$; Figure $1 \mathrm{~A}$, black trace). The magnitude of persistent current is lower than previous reports due to differences in cell type and recording conditions. ${ }^{10}$ To assess the ability of either GS967 or phenytoin to selectively inhibit persistent sodium current, neurons were acutely treated with drug concentrations approximating the therapeutic free drug concentration in mouse brain, $200 \mathrm{nmol} \cdot \mathrm{L}^{-1}$ GS967 or $4 \mu \mathrm{mol} \cdot \mathrm{L}^{-1}$ phenytoin. In the presence of GS967, persistent current was $>10$-fold lower than in the absence of compound $(0.12 \pm 0.06 \%$ of peak current, $\mathrm{n}=7 ; P<.001$, 1-way ANOVA and Tukey post hoc). In the presence of phenytoin, persistent current was approximately 2-fold lower than in drug-free conditions $(0.94 \pm 0.09 \%$ of peak current, $\mathrm{n}=6 ; P<.04)$. Thus, GS967 was more potent than phenytoin in suppressing persistent current mediated by the mutant channel $(P<.04$; Figure 1A,B). Importantly, neither compound significantly altered peak sodium current under these conditions (Figure 1C).

\section{2 | GS967 suppresses EADs and reduces neuronal excitability in CA1 pyramidal neurons from $\mathrm{Scn} 8 \mathrm{a}^{\mathrm{D/+}}$ mice}

AP waveforms from $S c n 8 a^{D /+}$ mouse CA1 pyramidal neurons exhibited prominent EADs that were evident during the repolarization phase of APs (Figure 2A-C), in agreement with previous studies. ${ }^{30}$ These abnormal waveforms were not observed in WT controls (Figure 2D). Addition of GS967 had a profound effect on EADs, reducing their number by $41 \%$ at $500 \mathrm{nmol} \cdot \mathrm{L}^{-1}$ and $50 \%$ at $1 \mu \mathrm{mol} \cdot \mathrm{L}^{-1} \mathrm{GS} 967$ (Figure 2B,C). GS967 had no effect on AP firing frequencies from either $S c n 8 a^{D /+}$ or WT (+/+) neurons (Figure 2Ai-Di). Analysis of AP parameters revealed differences between Scn $8 a^{D /+}$ and WT neurons (Table S1). GS967 (1 $\left.\mu \mathrm{mol} \cdot \mathrm{L}^{-1}\right)$ decreased AP amplitude and upstroke velocity in both $\operatorname{Scn} 8 a^{D /+}$ and WT neurons. AP thresholds were also depolarized in $S c n 8 a^{D /+}$ neurons, but not WT (Figure 2E, Table S1). APDs were not changed (Table S1). These findings demonstrate that GS967 can modulate intrinsic hyperexcitability of CA1 neurons from $S c n 8 a^{D /+}$ mice.

\subsection{GS967 is more potent than phenytoin for seizure protection in the MES assay}

We assessed acute anticonvulsant activity of GS967 and phenytoin using the MES paradigm in $\operatorname{Scn} 8 a^{D /+}$ mice and
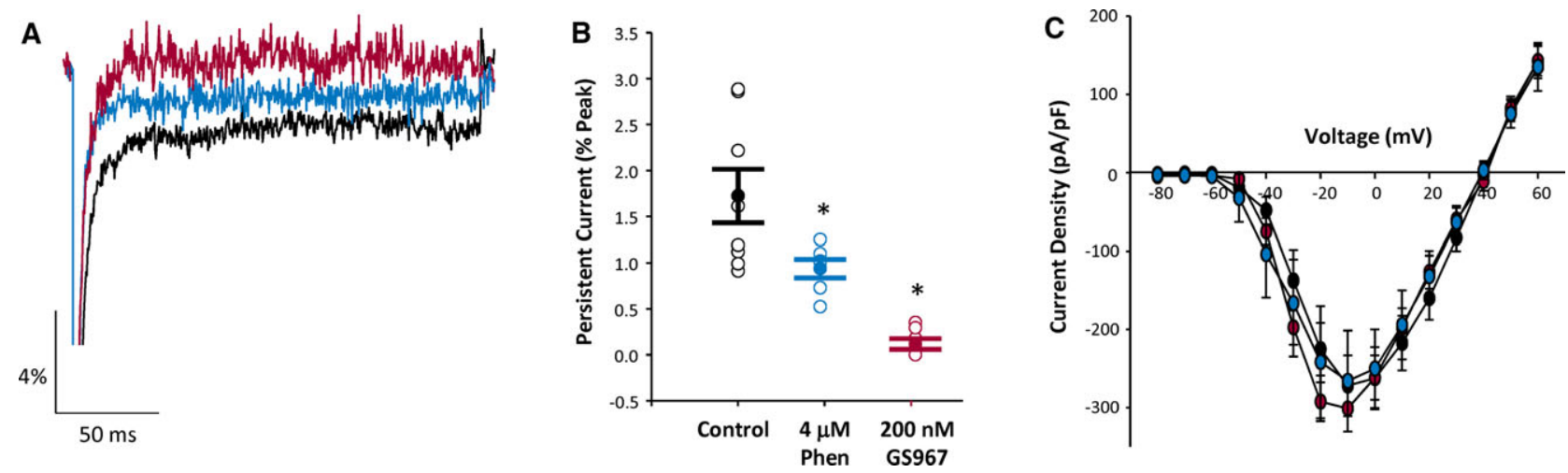

F I G URE 1 GS967 inhibits persistent sodium current in acutely isolated hippocampal pyramidal neurons from $S c n 8 a^{D /+}$ mice.

A, Representative traces showing persistent sodium current of untreated (black), GS967-treated (red), and phenytoin (Phen)-treated (blue) neurons from $S c n 8 a^{D /+}$ mice. B,C, Summary data of persistent sodium current (B) and current-voltage relationship for peak sodium current (C) for untreated (black symbols, $\mathrm{n}=8$ ), phenytoin-treated (blue symbols, $\mathrm{n}=6$ ), and GS967-treated (red symbols, $\mathrm{n}=7$ ) neurons from $S c n 8 a^{D /+}$ mice. Open symbols represent individual cells, whereas closed symbols represent mean \pm standard error of the mean. Statistical comparison was performed using 1-way analysis of variance followed by Tukey's post hoc test. *Statistically significant difference compared to untreated cells for phenytoin-treated $(P<.04)$ and GS967-treated $(P<.001)$ cells 

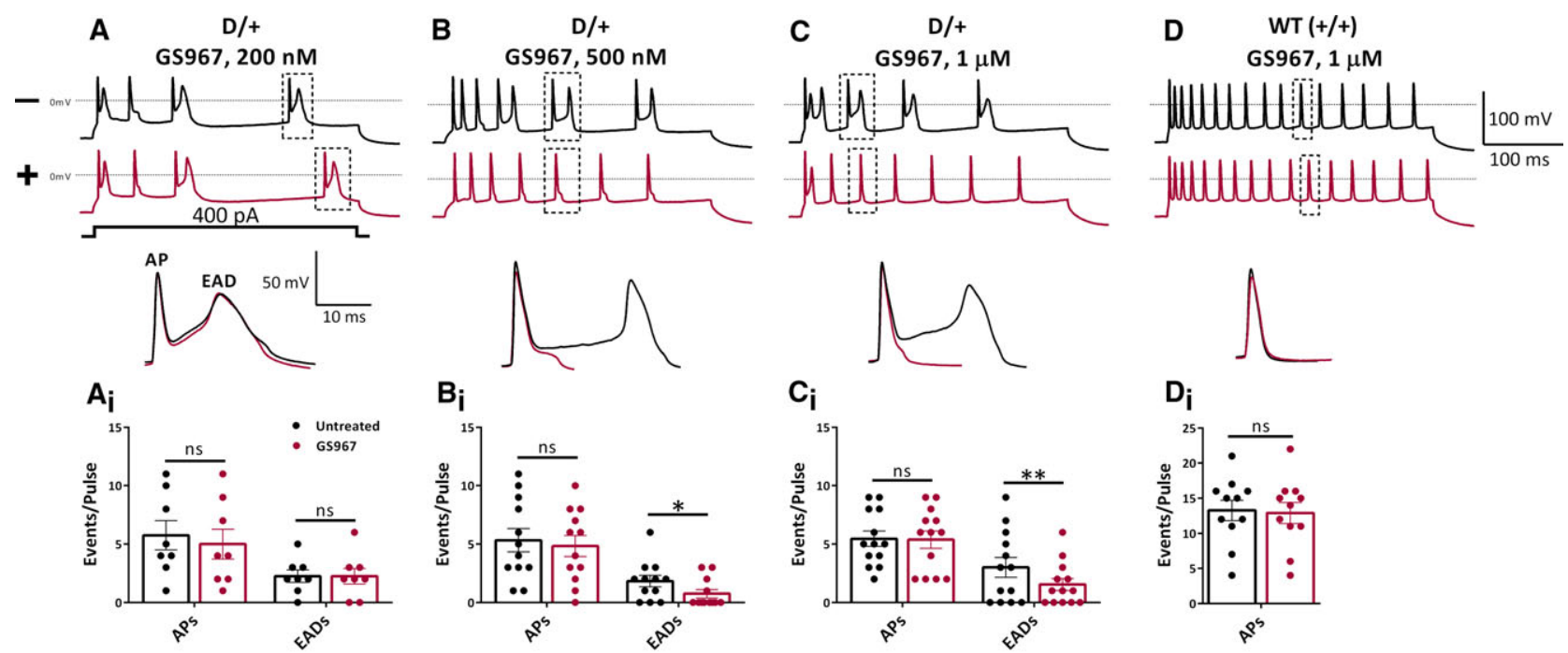

E

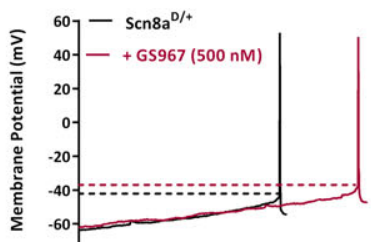

FIGURE 2 GS967 suppresses early afterdepolarizations (EADs) and reduces intrinsic excitability of CA1 pyramidal neurons from $S c n 8 a^{D /+}$ mice. A-D, Upper panel traces show representative action potentials (APs) evoked using a 400-pA depolarizing current injection step. Black indicates before GS967; red indicates after GS967 at (A) $200 \mathrm{nmol} \cdot \mathrm{L}^{-1}$ ( $\mathrm{n}=8$ from 3 mice), (B) $500 \mathrm{nmol} \cdot \mathrm{L}^{-1}$ ( $\mathrm{n}=12 \mathrm{from} 3 \mathrm{mice}$ ), and (C) $1 \mu \mathrm{mol} \cdot \mathrm{L}^{-1}(\mathrm{n}=13 \mathrm{from} 7 \mathrm{mice})$; (D) $1 \mathrm{lmolL}^{-1}$ in wild-type $(\mathrm{WT} ;+/+)(\mathrm{n}=11$ from 3 mice). Ai-Di, Lower panels show representative EADs and bar/scatter plots showing effect of GS967 on APs and EADs evoked by a current injection step of 400 pA. E, Representative trace for slow current ramp recorded from a CA1 pyramidal neuron before (black) and after (red) GS967 at $500 \mathrm{nmol} \cdot \mathrm{L}^{-1}$. Values are mean \pm standard error of the mean. Significant results were determined using paired Student's $t$ test with alpha set to .05 . ns, nonsignificant. $* P<.05$, $* * P<.01$

WT littermates. The electroconvulsive stimulus (ECS) required to induce maximal hindlimb extension in $>95 \%$ of mice $\left(\mathrm{ECS}_{95}\right)$ was determined for both genotypes. $\operatorname{Scn} 8 a^{D /+}$ mice required a stimulus of $120 \mathrm{mC}$ compared to $720 \mathrm{mC}$ for WT mice. Varying doses of GS967 or phenytoin were administered 2 hours prior to MES induction using the genotype-appropriate $\mathrm{ECS}_{95}$. Both GS967 and phenytoin provided dose-dependent protection against MES-induced tonic hindlimb seizures, with GS967 exhibiting greater potency. $\operatorname{Scn} 8 a^{D /+}$ mice were protected from maximal hindlimb extension with a calculated $\mathrm{ED}_{50}$ of $1.2 \pm 0.2 \mathrm{mg} / \mathrm{kg}$ for GS967 and $5.3 \pm 0.7 \mathrm{mg} / \mathrm{kg}$ for phenytoin (Figure 3A). Similar results were found in WT mice with an $\mathrm{ED}_{50}$ of $0.7 \pm 0.2 \mathrm{mg} / \mathrm{kg}$ for GS967 and $6.9 \pm 0.5 \mathrm{mg} / \mathrm{kg}$ for phenytoin (Figure 3B).

\subsection{Chronic GS967 treatment prolongs survival of $S c n 8 a^{D /+}$ mice}

Survival of $\operatorname{Scn} 8 a^{D /+}$ mice is limited, with $<50 \%$ of mice surviving to 6 months of age. ${ }^{9}$ To determine whether chronic treatment with GS967 would extend survival, $\operatorname{Scn} 8 a^{D /+}$ mice were fed chow containing GS967 (estimated dose $=1.5 \mathrm{mg} / \mathrm{kg} / \mathrm{d}$ ) beginning at 6 weeks and continuing until 6 months of age. This dose was previously shown to be anticonvulsant in Scn2a $a^{Q 54}$ and Scnla ${ }^{+/-}$mouse mod$\mathrm{els}^{23,25}$ and does not produce any signs of behavioral toxicity or sedation (Figure S1). Treatment with GS967 beginning at 6 weeks and continuing to 6 months of age completely rescued premature lethality of $S c n 8 a^{D /+}$ mice, with $100 \%$ of GS967-treated mice $(\mathrm{n}=32)$ surviving to 6 months of age, compared with only $24 \%$ of untreated mice (7/29; $P<.001$, Mantel-Cox log-rank). Removal of the GS967 chow at 6 months of age resulted in rapid loss of protection, and $65 \%$ of the previously treated mice died within the following 3 months (Figure 4A). Loss of GS967 benefit following withdrawal suggests that it acts primarily as an anticonvulsant rather than a disease-modifying treatment.

The phenotype of homozygous $\operatorname{Scn} 8 a^{D / D}$ mice is much more severe than heterozygous $S c n 8 a^{D /+}$ mice or heterozygous patients. $S c n 8 a^{D / D}$ mice exhibit $100 \%$ lethality by 21 days of age. ${ }^{9}$ Treatment of $S c n 8 a^{D / D}$ mice, achieved by feeding nursing dams GS967 beginning at P5, extended survival by 1 week $(P<.001$, Mantel-Cox log-rank; Figure 4B). 

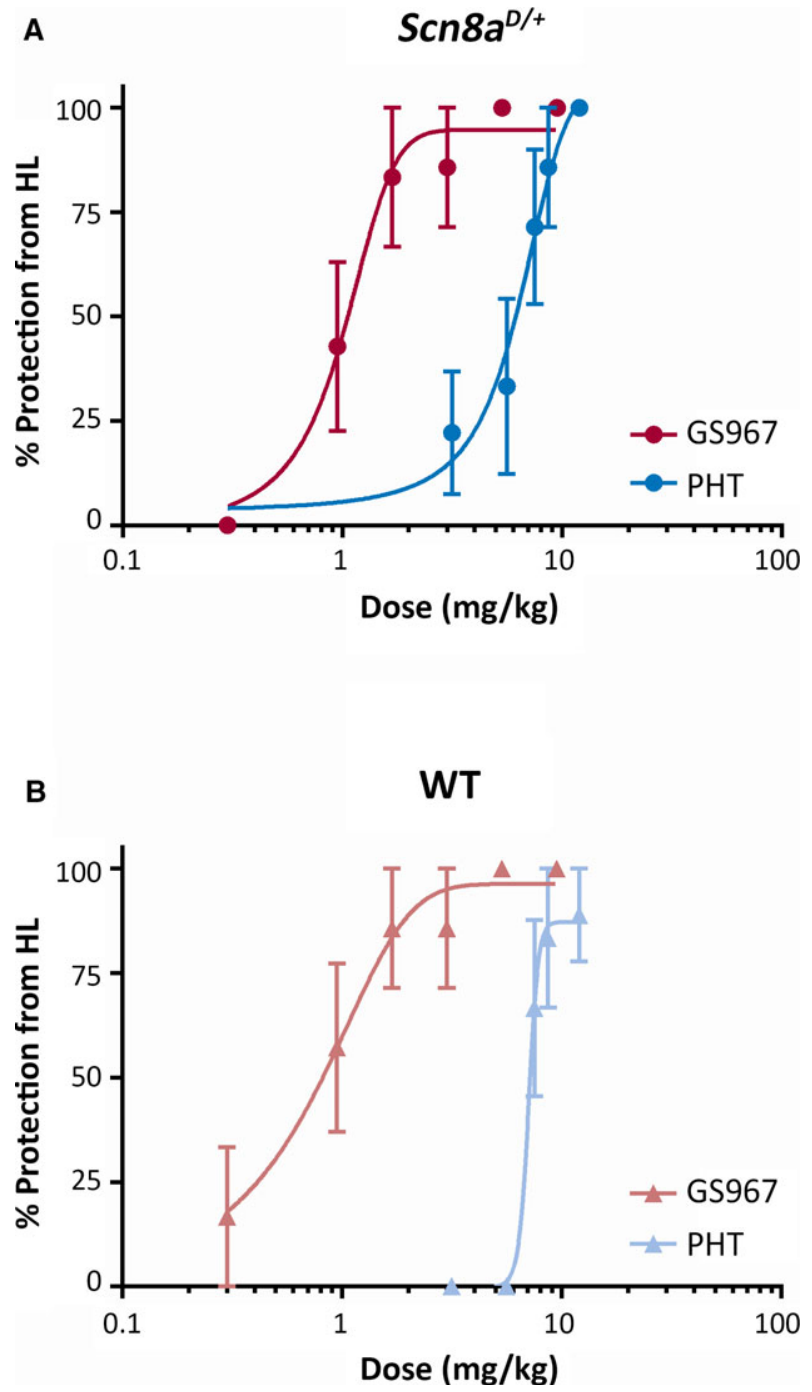

FIGURE 3 GS967 protects against maximal electroshock (MES)-induced seizures in $S c n 8 a^{D /+}$ and wild-type (WT) mice with greater potency than phenytoin (PHT). Mice were pretreated with test compound or vehicle and then received an electroconvulsive stimulus determined to reliably elicit seizures with maximal hindlimb extension in $S c n 8 a^{D /+}(120 \mathrm{mC})$ or WT $(720 \mathrm{mC})$ mice. A, Dose-response curves of GS967 and phenytoin for MES seizures in $S c n 8 a^{D /+}$ mice. The curve for GS967 is significantly shifted compared to phenytoin, and the estimated $\mathrm{ED}_{50}$ for GS967 and phenytoin are $1.1 \pm 0.23$ and $5.33 \pm 0.69 \mathrm{mg} / \mathrm{kg}$, respectively $(P<.001, \mathrm{n}=5-24$, Student's $t$ test). Symbols represent mean \pm standard error of the mean. B, Doseresponse curves of GS967 and phenytoin for MES seizures in WT mice. The curve is also significantly shifted, and the estimated $\mathrm{ED}_{50}$ for GS967 and phenytoin are $0.70 \pm 0.22$ and $6.93 \pm 0.45 \mathrm{mg} / \mathrm{kg}$, respectively $(P<.001, \mathrm{n}=6-26$, Student's $t$ test $)$

\subsection{GS967 reduces spontaneous seizures in $\mathrm{Scn} 8 \mathrm{a}^{\mathrm{D/+}}$ mice}

Previous reports showed that $S c n 8 a^{D /+}$ mice have spontaneous seizures with onset at 2 to 4 months of age, followed by death within a few days of seizure onset. ${ }^{9,31}$ To evaluate the effect of GS967 on spontaneous seizures, mice were continuously observed with a combination of video-EEG monitoring and continuous video monitoring for several hundred hours (Figure 5; Figure S2). Only 2 of 7 GS967-treated mice experienced any seizures during the time of monitoring, compared with 7 of 7 untreated mice (Table 1). All recorded seizures in $S c n 8 a^{D /+}$ mice initiated with a tonic phase and culminated in full tonic hindlimb extension, with the angle of hindlimbs to the torso $\geq 180^{\circ}$. Several of the untreated mice exhibited seizure clustering, with days of seizure freedom separating periods of high seizure frequency (Figure S2). Untreated $S c n 8 a^{D /+}$ mice also exhibited seizures with multiple tonic to tonic-clonic transitions. In contrast, seizures in the GS967-treated $S c n 8 a^{D /+}$ mice had a single tonic phase that terminated with postictal suppression. Combined analysis of video-EEG and video monitoring showed that Scn $8 a^{D /+}$ mice treated with GS967 had significantly lower seizure frequency, with $0.3 \pm 0.2$ seizures/ 24 hours in GS967-treated compared with $1.6 \pm 0.4$ seizures/24 hours in untreated mice $(P<.004$, Figure 5C, Table 1$)$. No seizures were observed in WT littermate mice (Table 1). These data demonstrate that GS967 has a potent antiseizure effect in a mouse model of SCN8A encephalopathy.

\section{4 | DISCUSSION}

In this study, we demonstrated that GS967 significantly reduced spontaneous seizure burden and improved survival in the $S c n 8 a^{D /+}$ epilepsy model. We also demonstrated that GS967 provided protection from acute MES-induced seizures in both $\operatorname{Scn} 8 a^{D /+}$ and WT mice.

\subsection{GS967 is a potent blocker of persistent current arising from the Scn8 $a^{N 1768 D}$ mutation}

We and others previously reported that GS967 is a potent inhibitor of persistent sodium current, ${ }^{22,24,32-34}$ with 9-fold greater potency than phenytoin in hippocampal pyramidal neurons from $\operatorname{Scn} 2 a^{Q 54}$ mice. ${ }^{25}$ Consistent with these reports, acute application of $200 \mathrm{nmol} \cdot \mathrm{L}^{-1} \mathrm{GS} 967$ or $4 \mu \mathrm{mol} \cdot \mathrm{L}^{-1}$ phenytoin to acutely dissociated hippocampal neurons isolated from $\operatorname{Scn} 8 a^{D /+}$ mice resulted in inhibition of persistent sodium current without significantly affecting peak current. Similarly, acute application of GS967 to hippocampal slices from $\operatorname{Scn} 8 a^{D /+}$ mice resulted in normalization of intrinsic excitability in CA1 neurons.

\subsection{Anticonvulsant activity of GS967}

To assess anticonvulsant activity of GS967, we first established the dose-response relationship of acutely administered GS967 for MES-induced seizures, using the 

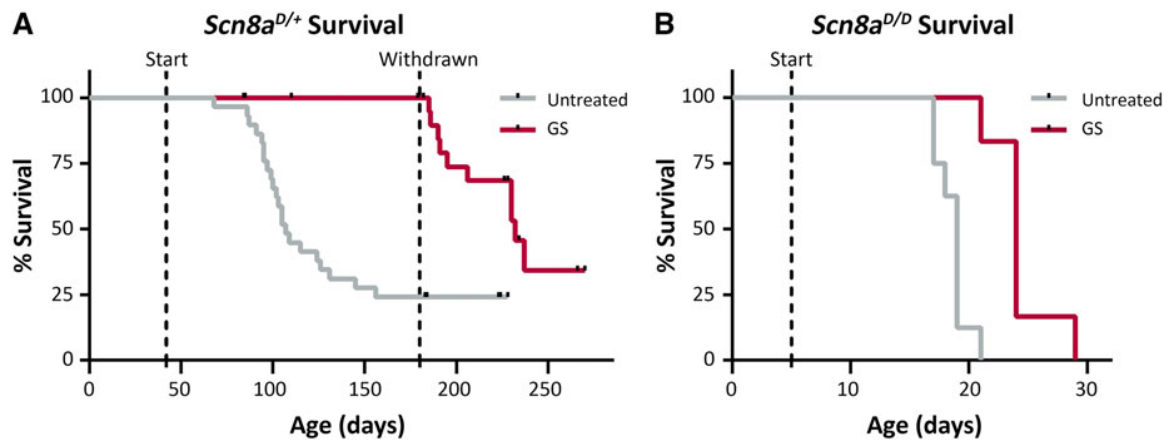

F I G U RE 4 Treatment with GS967 (GS) significantly prolongs survival in heterozygous and homozygous mutants. A, Kaplan-Meier survival curves comparing untreated and GS967-treated $\operatorname{Scn} 8 a^{D /+}$ mice. Treatment was started at 6 weeks of age (first dashed line) and was withdrawn at 6 months (second dashed line). Survival was significantly different between treatment groups $(P<.001 ; \mathrm{n}=29-32$; Mantel-Cox log-rank test). B, Kaplan-Meier survival curves comparing untreated and GS967-treated $S c n 8 a^{D / D}$ mice. Treatment was started at 5 days of age (dashed line). Survival difference between treatment groups was significant $(P<.001 ; \mathrm{n}=6$-8; Mantel-Cox log-rank test). WT, wild-type

conventional anticonvulsant phenytoin as a comparator. We demonstrated that GS967 provided dose-dependent protection against MES-induced seizures with $\sim 5-10$-fold greater potency than phenytoin in both WT and $\operatorname{Scn} 8 a^{D /+}$ mice. Chronic administration of GS967 was associated with a dramatically lower spontaneous seizure frequency and prevented lethality at a dose that did not cause overt neurobehavioral toxicity or sedation. GS967 treatment provided complete protection in heterozygous $\operatorname{Scn} 8 a^{D /+}$ mice that have the same genotype as the N1768D proband with SCN8A encephalopathy, resulting from a heterozygous de novo mutation. Even in the severely affected homozygous
$\operatorname{Scn} 8 a^{D / D}$ mice, GS967 extended survival from 3 to 4 weeks of age. Similar to individuals with SCN8A encephalopathy, $S c n 8 a^{D /+}$ mice exhibit tonic and/or tonicclonic seizures that sometimes occur in clusters. VideoEEG monitoring showed that untreated $S c n 8 a^{D /+}$ mice often exhibit prolonged seizures with multiple tonic to tonic-clonic transitions. GS967-treated mice were completely protected from these multiphase events and exhibited only isolated seizures with a single tonic phase. These observations suggest that GS967 may reduce the extent of ictal events, in addition to reducing seizure frequency. These effects are likely to contribute to the survival benefit,
F I G U RE 5 Treatment with GS967 reduces spontaneous seizure frequency in Scn $8 a^{D /+}$ mice. A, Schematic representation of timeline for treatment, surgery, and monitoring of mice. B, Representative electroencephalographic (EEG) trace of an electrographic seizure in an $\operatorname{Scn} 8 a^{D /+}$ mouse. Channel 1 indicates left anterior to right anterior; channel 2 indicates right anterior to left posterior. Upper panel, 5 min window. Lower panel, expanded timescale of seizure shown in upper panel. $\mathrm{C}$, Seizure incidence frequency was significantly lower in $S c n 8 a^{D /+}$ mice treated with GS967 compared to untreated mice $(P<.004$; Mann-Whitney test; $\mathrm{n}=7$ per group). Symbols represent mean \pm standard error of the mean

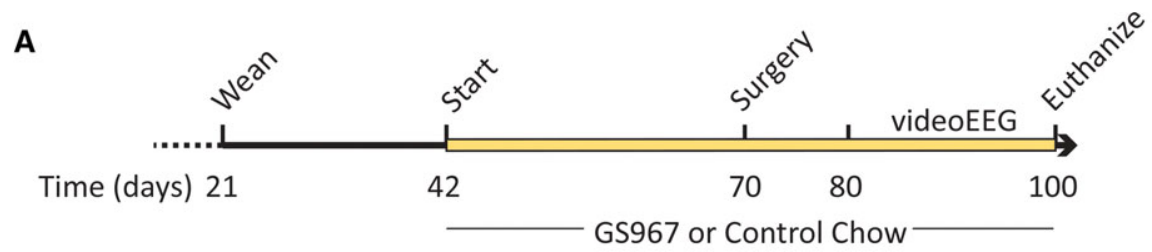

B
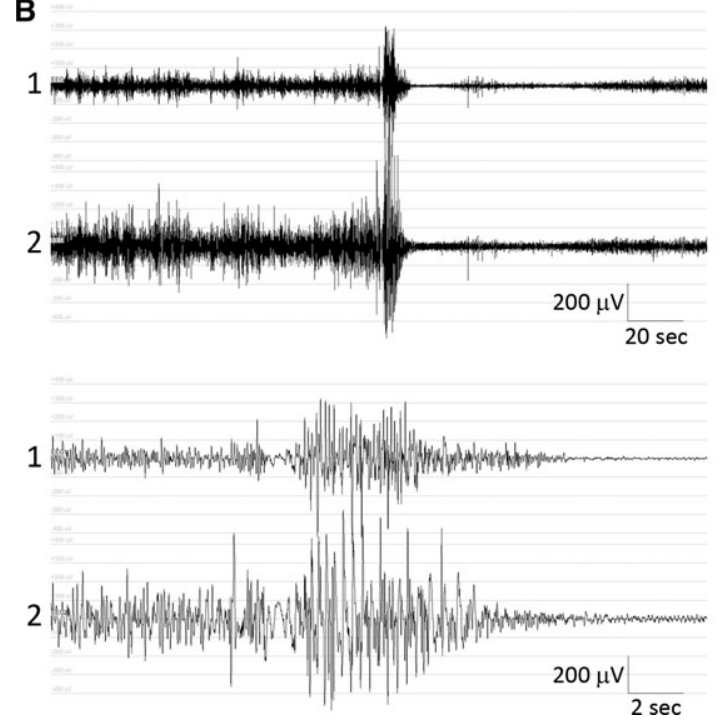

C

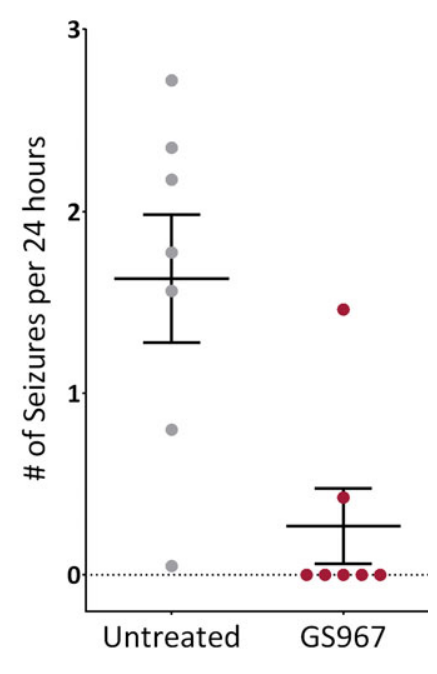


TABLE 1 Extended spontaneous seizure monitoring in $S c n 8 a^{D /+}$ and WT mice treated chronically with $1.5 \mathrm{mg} / \mathrm{kg} / \mathrm{d}$ GS967 and untreated controls

\begin{tabular}{|c|c|c|c|c|c|c|c|c|}
\hline Treatment & Genotype & $\mathbf{n}$ & $\begin{array}{l}\text { Total } \\
\text { hours } \\
\text { monitored }\end{array}$ & $\begin{array}{l}\text { Average hours } \\
\text { monitored per } \\
\text { animal } \pm \text { SEM (range) }\end{array}$ & $\begin{array}{l}\text { Total } \\
\text { seizures, } \\
\text { n }\end{array}$ & $\begin{array}{l}\text { Seizures with } \\
\text { multiple } \\
\text { transitions, \% }\end{array}$ & $\begin{array}{l}\text { Average } \\
\text { seizure } \\
\text { frequency }\end{array}$ & $\begin{array}{l}\text { Fraction of mice } \\
\text { with observed } \\
\text { seizures }\end{array}$ \\
\hline GS967 & $\operatorname{Scn} 8 a^{D /+}$ & 7 & 1699 & $243 \pm 52(62-361)$ & 9 & 0 & $0.3 \pm 0.2$ & $2 / 7$ \\
\hline Untreated & $\operatorname{Scn} 8 a^{D /+}$ & 7 & 2189 & $313 \pm 94(60-614)$ & 137 & 46 & $1.6 \pm 0.4$ & $7 / 7$ \\
\hline GS967 & WT & 3 & 628 & $209 \pm 172(102-408)$ & 0 & 0 & $0 \pm 0.0$ & $0 / 3$ \\
\hline
\end{tabular}

SEM, standard error of the mean; WT, wild-type.

${ }^{\mathrm{a}}$ Seizures per 24 hours \pm SEM, calculated by averaging individual seizure frequencies within each group.

because poor seizure control and frequent, prolonged generalized tonic-clonic seizures are major risk factors for SUDEP. ${ }^{35}$ GS967 was originally developed as an antiarrhythmic compound, ${ }^{22,24,32,33}$ and may also have a direct effect on the heart. This effect may contribute to the protection against sudden death, because $S c n 8 a^{D /+}$ mice have hyperexcitable cardiomyocytes, and episodes of bradycardia have been observed in electrocardiograms. ${ }^{36}$

Although we demonstrate dramatic improvement in seizure burden and survival in $\operatorname{Scn} 8 a^{D /+}$ mice treated with GS967, there are some limitations of our preclinical study that warrant consideration. First, GS967 treatment was commenced prior to seizure onset and it is not known whether commencing treatment after fulminant onset would provide the same level of protection. Second, the age of seizure onset in the mouse $\operatorname{Scn} 8 a^{D /+}$ model is later than that observed in the proband, suggesting that the mouse model does not directly phenocopy the human case. Third, SCN8A encephalopathy includes mutations that result in a variety of $\mathrm{Na}_{\mathrm{V}} 1.6$ dysfunction ranging from gain-of-function defects, including but not limited to persistent sodium current, to loss-of-function defects. This suggests that GS967 would not necessarily be broadly effective in all cases of SCN8A encephalopathy, but rather that knowledge of the specific channel dysfunction may provide additional information for rational therapy choice.

Beyond $\operatorname{Scn} 8 a^{D /+}$ mice, GS967 was previously shown to reduce seizure burden and improve survival in Scn $2 a^{Q 54}$ mice, which share the biophysical defect of elevated persistent sodium current. ${ }^{25,37}$ GS967 was also shown to have a protective effect in the Scnla ${ }^{+/}$Dravet mouse model that has reduced activity in inhibitory neurons and elevated excitability in excitatory neurons in mice. ${ }^{23,26}$ However, there is no evidence for alteration of persistent current in this model, suggesting that GS967 can have protective benefit in a genetic model with an alternate underlying mechanism. Furthermore, we demonstrated here and in a previous report that GS967 has potent antiseizure activity in the MES assay in WT mice, ${ }^{25}$ supporting potentially broader applicability of GS967 in epilepsies with divergent etiologies.

\section{CONCLUSIONS}

We demonstrate that targeting of a specific biophysical defect in $\mathrm{Na}_{\mathrm{V}} 1.6$ gating observed with some SCN8A patient mutations is an effective antiepileptic treatment strategy in $S c n 8 a^{D /+}$ mice. This supports a precision medicine approach to $S C N 8 A$ encephalopathy and suggests that defining the biophysical defect may provide valuable information to guide therapy. Moreover, results from acute MES studies and other genetic models suggest that suppression of $\mathrm{Na}_{\mathrm{V}} 1.6$ activity may also be a general therapeutic approach relevant to other genetic and nongenetic epilepsies.

\section{ACKNOWLEDGMENTS}

We thank Nicole Zachwieja and Tatiana Abramova for technical assistance, and Lyndsey Anderson for experimental guidance. This study was supported by Northwestern University Feinberg School of Medicine and National Institutes of Health grant R01 NS103090 (M.K.P.). E.M.B. is supported by a predoctoral fellowship from the PhRMA Foundation. Initial aliquots of GS967 were provided Gilead Sciences.

\section{DISCLOSURE OF CONFLICT OF INTEREST}

J.A.K. and A.L.G. have received support from and/or have served as a paid consultant for Praxis Precision Medicines. A.L.G. served as a paid consultant for Gilead Sciences. The remaining authors have no conflicts of interest. We confirm that we have read the Journal's position on issues involved in ethical publication and affirm that this report is consistent with those guidelines. 


\section{REFERENCES}

1. Hesdorffer DC, Logroscino G, Benn EK, et al. Estimating risk for developing epilepsy: a population-based study in Rochester, Minnesota. Neurology. 2011;76:23-7.

2. Meisler MH, Kearney JA. Sodium channel mutations in epilepsy and other neurological disorders. J Clin Invest. 2005;115:2010-7.

3. Scheffer IE, Berkovic S, Capovilla G, et al. ILAE classification of the epilepsies: position paper of the ILAE Commission for Classification and Terminology. Epilepsia. 2017;58:512-21.

4. Hammer MF, Wagnon JL, Mefford HC, et al. SCN8A-related epilepsy with encephalopathy. In: Pagon RA, Adam MP, Ardinger $\mathrm{HH}$, et al, editors. GeneReviews. Seattle, WA: University of Washington, Seattle; 2016.

5. Wagnon JL, Meisler MH. Recurrent and non-recurrent mutations of SCN8A in epileptic encephalopathy. Front Neurol. 2015;6:104.

6. Harden C, Tomson T, Gloss D, et al. Practice guideline summary: sudden unexpected death in epilepsy incidence rates and risk factors: report of the guideline development, dissemination, and implementation subcommittee of the American Academy of Neurology and the American Epilepsy Society. Neurology. 2017;88: 1674-80.

7. Veeramah KR, O'Brien JE, Meisler MH, et al. De novo pathogenic SCN8A mutation identified by whole-genome sequencing of a family quartet affected by infantile epileptic encephalopathy and SUDEP. Am J Hum Genet. 2012;90:502-10.

8. Jones JM, Meisler MH. Modeling human epilepsy by TALEN targeting of mouse sodium channel Scn8a. Genesis. 2014;52:141-8.

9. Wagnon JL, Korn MJ, Parent R, et al. Convulsive seizures and SUDEP in a mouse model of SCN8A epileptic encephalopathy. Hum Mol Genet. 2015;24:506-15.

10. Ottolini M, Barker BS, Gaykema RP, et al. Aberrant sodium channel currents and hyperexcitability of medial entorhinal cortex neurons in a mouse model of SCN8A encephalopathy. J Neurosci. 2017;37:7643-55.

11. Barker BS, Ottolini M, Wagnon JL, et al. The SCN8A encephalopathy mutation p.Ile1327Val displays elevated sensitivity to the anticonvulsant phenytoin. Epilepsia. 2016;57:1458-66.

12. Boerma RS, Braun KP, van den Broek MP, et al. Remarkable phenytoin sensitivity in 4 children with SCN8A-related epilepsy: a molecular neuropharmacological approach. Neurotherapeutics. 2016;13:192-7.

13. Braakman HM, Verhoeven JS, Erasmus CE, et al. Phenytoin as a last-resort treatment in SCN8A encephalopathy. Epilepsia Open. 2017;2:343-4.

14. Larsen J, Carvill GL, Gardella E, et al. The phenotypic spectrum of SCN8A encephalopathy. Neurology. 2015;84:480-9.

15. Meisler MH, Helman G, Hammer MF, et al. SCN8A encephalopathy: research progress and prospects. Epilepsia. 2016; 57:1027-35.

16. Wang J, Gao H, Bao X, et al. SCN8A mutations in Chinese patients with early onset epileptic encephalopathy and benign infantile seizures. BMC Med Genet. 2017;18:104.

17. Segal MM, Douglas AF. Late sodium channel openings underlying epileptiform activity are preferentially diminished by the anticonvulsant phenytoin. J Neurophysiol. 1997;77:3021-34.

18. Imam SH, Landry K, Kaul V, et al. Free phenytoin toxicity. Am J Emerg Med. 2014;32:1301.e3-4.
19. Marin LL, Garcia-Penas JJ, Herguedas JL, et al. Phenytoininduced visual disturbances mimicking delirium tremens in a child. Eur J Paediatr Neurol. 2010;14:460-3.

20. Phelps SJ, Baldree LA, Boucher BA, et al. Neuropsychiatric toxicity of phenytoin. Importance of monitoring phenytoin levels. Clin Pediatr (Phila). 1993;32:107-10.

21. Robertson K, von Stempel CB, Arnold I. When less is more: a case of phenytoin toxicity. BMJ Case Rep. 2013;2013:pii: bcr2012008023.

22. Belardinelli L, Liu G, Smith-Maxwell C, et al. A novel, potent, and selective inhibitor of cardiac late sodium current suppresses experimental arrhythmias. J Pharmacol Exp Ther. 2013;344:2332.

23. Anderson LL, Hawkins NA, Thompson $\mathrm{CH}$, et al. Unexpected efficacy of a novel sodium channel modulator in Dravet syndrome. Sci Rep. 2017;7:1682.

24. Potet F, Vanoye CG, George AL Jr. Use-dependent block of human cardiac sodium channels by GS967. Mol Pharmacol. 2016;90:52-60.

25. Anderson LL, Thompson CH, Hawkins NA, et al. Antiepileptic activity of preferential inhibitors of persistent sodium current. Epilepsia. 2014;55:1274-83.

26. Mistry AM, Thompson $\mathrm{CH}$, Miller AR, et al. Strain- and agedependent hippocampal neuron sodium currents correlate with epilepsy severity in Dravet syndrome mice. Neurobiol Dis. 2014;65:1-11.

27. Thompson CH, Hawkins NA, Kearney JA, et al. CaMKII modulates sodium current in neurons from epileptic Scn2a mutant mice. Proc Natl Acad Sci U S A. 2017;114:1696-701.

28. Yamada-Hanff J, Bean BP. Persistent sodium current drives conditional pacemaking in CA1 pyramidal neurons under muscarinic stimulation. J Neurosci. 2013;33:15011-21.

29. Irwin S. Comprehensive observational assessment: Ia. A systematic, quantitative procedure for assessing the behavioral and physiologic state of the mouse. Psychopharmacologia. 1968;13:22257.

30. Lopez-Santiago LF, Yuan Y, Wagnon JL, et al. Neuronal hyperexcitability in a mouse model of SCN8A epileptic encephalopathy. Proc Natl Acad Sci U S A. 2017;114:2383-8.

31. Sprissler RS, Wagnon JL, Bunton-Stasyshyn RK, et al. Altered gene expression profile in a mouse model of SCN8A encephalopathy. Exp Neurol. 2017;288:134-41.

32. Pezhouman A, Madahian S, Stepanyan H, et al. Selective inhibition of late sodium current suppresses ventricular tachycardia and fibrillation in intact rat hearts. Heart Rhythm. 2014;11:492-501.

33. Sicouri S, Belardinelli L, Antzelevitch C. Antiarrhythmic effects of the highly selective late sodium channel current blocker GS458967. Heart Rhythm. 2013;10:1036-43.

34. Hirakawa R, El-Bizri N, Shryock JC, et al. Block of Na+ currents and suppression of action potentials in embryonic rat dorsal root ganglion neurons by ranolazine. Neuropharmacology. 2012;62:2251-60.

35. Lhatoo S, Noebels J, Whittemore V, et al. Sudden unexpected death in epilepsy: identifying risk and preventing mortality. Epilepsia. 2015;56:1700-6.

36. Frasier CR, Wagnon JL, Bao YO, et al. Cardiac arrhythmia in a mouse model of sodium channel SCN8A epileptic encephalopathy. Proc Natl Acad Sci U S A. 2016;113:12838-43. 
37. Kearney JA, Plummer NW, Smith MR, et al. A gain-of-function mutation in the sodium channel gene $\operatorname{Sen} 2 \mathrm{a}$ results in seizures and behavioral abnormalities. Neuroscience. 2001;102:307-17.

\section{SUPPORTING INFORMATION}

Additional supporting information may be found online in the Supporting Information section at the end of the article.
How to cite this article: Baker EM, Thompson $\mathrm{CH}$, Hawkins NA, et al. The novel sodium channel modulator GS-458967 (GS967) is an effective treatment in a mouse model of $S C N 8 A$ encephalopathy. Epilepsia. 2018;59:1166-1176. https://doi.org/10.1111/epi.14196 